\title{
Front Matter: Volume 7365
}

, "Front Matter: Volume 7365," Proc. SPIE 7365, Bioengineered and Bioinspired Systems IV, 736501 (22 June 2009); doi: 10.1117/12.834637

SPIE Event: SPIE Europe Microtechnologies for the New Millennium, 2009, SPIE. Dresden, Germany 


\title{
PROCEEDINGS OF SPIE
}

\section{Bioengineered and Bioinspired Systems IV}

\author{
Ángel B. Rodríguez-Vázquez \\ Ricardo A. Carmona-Galán \\ Gustavo Liñán-Cembrano \\ Editors
}

4-6 May 2009

Dresden, Germany

Sponsored by

SPIE Europe

GMM VDE/VDI Gesellschaft Mikroelektronik, Mikro- und Feinmechanik

Cooperating Organizations

SPIE

Nanotechnology CC "Ultrathin functional films"

mst|news

Silicon Saxony

Published by

SPIE 
The papers included in this volume were part of the technical conference cited on the cover and title page. Papers were selected and subject to review by the editors and conference program committee. Some conference presentations may not be available for publication. The papers published in these proceedings reflect the work and thoughts of the authors and are published herein as submitted. The publisher is not responsible for the validity of the information or for any outcomes resulting from reliance thereon.

Please use the following format to cite material from this book:

Author(s), "Title of Paper," in Bioengineered and Bioinspired Systems IV, edited by Ángel B. Rodríguez-Vázquez, Ricardo A. Carmona-Galán, Gustavo Liñán-Cembrano, Proceedings of SPIE Vol. 7365 (SPIE, Bellingham, WA, 2009) Article CID Number.

ISSN 0277-786X

ISBN 9780819476395

Published by

SPIE

P.O. Box 10, Bellingham, Washington 98227-0010 USA

Telephone +1 3606763290 (Pacific Time) · Fax +1 3606471445

SPIE.org

Copyright (C) 2009, Society of Photo-Optical Instrumentation Engineers

Copying of material in this book for internal or personal use, or for the internal or personal use of specific clients, beyond the fair use provisions granted by the U.S. Copyright Law is authorized by SPIE subject to payment of copying fees. The Transactional Reporting Service base fee for this volume is $\$ 18.00$ per article (or portion thereof), which should be paid directly to the Copyright Clearance Center (CCC), 222 Rosewood Drive, Danvers, MA 01923. Payment may also be made electronically through CCC Online at copyright.com. Other copying for republication, resale, advertising or promotion, or any form of systematic or multiple reproduction of any material in this book is prohibited except with permission in writing from the publisher. The CCC fee code is 0277-786X/09/ $\$ 18.00$.

Printed in the United States of America.

Publication of record for individual papers is online in the SPIE Digital Library.

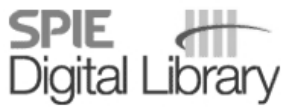

SPIEDigitalLibrary.org

Paper Numbering: Proceedings of SPIE follow an e-First publication model, with papers published first online and then in print and on CD-ROM. Papers are published as they are submitted and meet publication criteria. A unique, consistent, permanent citation identifier (CID) number is assigned to each article at the time of the first publication. Utilization of CIDs allows articles to be fully citable as soon they are published online, and connects the same identifier to all online, print, and electronic versions of the publication. SPIE uses a six-digit CID article numbering system in which:

- The first four digits correspond to the SPIE volume number.

- The last two digits indicate publication order within the volume using a Base 36 numbering system employing both numerals and letters. These two-number sets start with 00, 01, 02, 03, 04, $05,06,07,08,09,0 A, 0 B \ldots 0 Z$, followed by 10-1Z, 20-2Z, etc.

The CID number appears on each page of the manuscript. The complete citation is used on the first page, and an abbreviated version on subsequent pages. Numbers in the index correspond to the last two digits of the six-digit CID number. 


\section{Contents}

vii Conference Committee

SESSION 1 BIOINSPIRED VISION CHIPS AND ALGORITHMS

736502 Low-power focal-plane dynamic texture segmentation based on programmable image binning and diffusion hardware [7365-01]

J. Fernández-Berni, R. Carmona-Galán, CSIC, Univ. de Sevilla (Spain)

736503 A hierarchical artificial retina architecture [7365-02]

A. C. Parker, A. N. Azar, Univ. of Southern California (United States)

736504 Programmable active pixel sensor to investigate neural interactions within the retina [7365-03]

M. D. Hart, M. L. Prydderch, Science and Technology Research Council, Rutherford Appleton Lab. (United Kingdom); J. D. Morrison, D. Murdoch, K. Mathieson, Univ. of Glasgow (United Kingdom)

736505 Implementation of a new segmentation algorithm using the Eye-RIS CMOS vision system [7365-04]

F. Karabiber, Istanbul Univ. (Turkey); P. Arena, S. De Fiore, G. Vagliasindi, L. Fortuna, Univ. degli Studi di Catania (Italy); S. Arik, Istanbul Univ. (Turkey)

\section{SESSION 2 BIOINSPIRED ROBOT CONTROL AND NAVIGATION}

736506 STDP-based behavior learning on the TriBot robot [7365-05]

P. Arena, S. De Fiore, L. Patané, M. Pollino, C. Ventura, Univ. degli Studi di Catania (Italy)

736507 A new bio-inspired perceptual control architecture applied to solving navigation tasks [7365-06]

P. Arena, S. De Fiore, L. Patané, A. Vitanza, Univ. degli Studi di Catania (Italy)

736508 Path planning on cellular nonlinear network using active wave computing technique [7365-07]

R. Yeniçeri, M. E. Yalçın, Istanbul Technical Univ. (Turkey)

736509 Embedding the AnaFocus' Eye-RIS vision system in roving robots to enhance the action-oriented perception [7365-08]

L. Alba Soto, S. Morillas, J. Listán, A. Jiménez, AnaFocus (Spain); P. Arena, L. Patané,

S. De Fiore, Univ. degli Studi di Catania (Italy) 
7365 OA The infrared sensilla in the beetle Melanophila acuminata as model for new infrared sensors [7365-09]

H. Schmitz, Univ. Bonn (Germany); V. Norkus, Technische Univ. Dresden (Germany); N. Hess, DIAS Infrared GmbH (Germany); H. Bousack, Forschungszentrum Jülich GmbH (Germany)

$7365 \mathrm{OB} \quad$ Electric fish as natural models for technical sensor systems [7365-10]

G. von der Emde, Univ. Bonn (Germany); H. Bousack, C. Huck, K. Mayekar, M. Pabst, Y. Zhang, Forschungszentrum Jülich GmbH (Germany)

7365 OC Direct interfaces for smart skins based on FPGAs [7365-11]

Ó. Oballe-Peinado, J. Castellanos-Ramos, J. A. Hidalgo-López, F. Vidal-Verdú, Univ. de Málaga (Spain)

7365 OD Bio-inspired tactile sensing arrays [7365-12]

R. S. Dahiya, Italian Institute of Technology (Italy); M. Valle, Univ. degli Studi di Genova (Italy); G. Metta, Italian Institute of Technology (Italy) and Univ. degli Studi di Genova (Italy); L. Lorenzelli, Fondazione Bruno Kessler (Italy)

\section{SESSION 4 BIOMEDICAL APPLICATIONS AND DEVICES}

$7365 \mathrm{OE} \quad$ Spatio-temporal analysis of brain electrical activity in epilepsy based on cellular nonlinear networks [7365-13]

F. Gollas, Johann Wolfgang Goethe-Univ. Frankfurt am Main (Germany); R. Tetzlaff, Technische Univ. Dresden (Germany)

7365 OF Control electronics in an endoscopic capsule with locomotive, sensing, and actuating capabilities [7365-14]

O. Alonso, L. Freixas, J. Samitier, A. Dieguez, Univ. de Barcelona (Spain)

\section{SESSION 5 BIOCOMPATIBLE MATERIALS FABRICATION AND TECHNOLOGIES}

$7365 \mathrm{OH} \quad$ Direct fabrication of surface architectures on polymers and copolymers using laser interference patterning [7365-17]

A. Lasagni, Fraunhofer-Institut für Werkstoff- und Strahltechnik (Germany); D. Acevedo, Univ. Nacional de Río Cuarto (Argentina); M. Cornejo, Leibniz-Institut für Oberflächenmodifizierung e.V. (Germany); F. Lasagni, Fundación Andaluza de Desarrollo Aeronáutico (Spain); M. Politano, C. Barbero, Univ. Nacional de Río Cuarto (Argentina); F. Mücklich, Univ. des Saarlandes (Germany)

$7365 \mathrm{OI} \quad$ Rapid fabrication of biocompatible hydrogels microdevices using laser interference lithography [7365-18]

A. Lasagni, Georgia Institute of Technology (United States) and Fraunhofer-Institut für Werkstoff- und Strahltechnik (Germany); D. Yuan, P. Shao, S. Das, Georgia Institute of Technology (United States)

$73650 \mathrm{~J} \quad$ Lensless microscope using wavelength multiplexing [7365-19]

D. Fixler, A. Schwarz, Bar-llan Univ. (Israel); J. Garcia, Univ. de Valencia (Spain); Z. Zalevsky, Bar-llan Univ. (Israel) 
7365 OK Novel method for a flexible double-sided microelectrode fabrication process [7365-20] T. Doerge, S. Kammer, M. Hanauer, A. Sossalla, S. Steltenkamp, Fraunhofer-Institut für Biomedizinische Technik (Germany)

\section{SESSION $6 \quad$ NEURAL NETWORKS AND SOFT COMPUTING}

$73650 \mathrm{M}$ Token-passing communication protocol in hardware-based real-time spiking neural networks [7365-23]

B. Belhadj, J. Tomas, O. Malot, Y. Bornat, G. N'Kaoua, S. Renaud, Univ. Bordeaux 1 (France)

\section{SESSION 7 MICROFLUIDICS AND LAB-ON-A-CHIP}

736500 Sorting of solid and soft objects in vortices driven by surface acoustic waves [7365-25]

T. Franke, S. Braunmüller, T. Frommelt, A. Wixforth, Univ. Augsburg (Germany)

7365 OP Characterization of novel micro fluidic approaches for increased sensitivity of sensors measuring surface reactions [7365-26]

F. Sonntag, M. Gruchow, F. Mehringer, Fraunhofer Institut für Werkstoff- und Strahltechnik (Germany); S. Vogelsang, Technische Univ. Dresden (Germany); U. Klotzbach, E. Beyer, Fraunhofer Institut für Werkstoff- und Strahltechnik (Germany)

$73650 Q \quad$ Novel lab-on-a-chip system for the label-free detection of DNA hybridization and protein-protein interaction by surface plasmon resonance (SPR) [7365-27]

F. Sonntag, S. Schmieder, Fraunhofer Institut für Werkstoff- und Strahltechnik (Germany); N. Danz, Fraunhofer-Institut für Angewandte Optik und Feinmechanik (Germany); M. Mertig, Technische Univ. Dresden (Germany); N. Schilling, U. Klotzbach, E. Beyer, Fraunhofer Institut für Werkstoff- und Strahltechnik (Germany) 
Downloaded From: https://www.spiedigitallibrary.org/conference-proceedings-of-spie on 26 Apr 2023

Terms of Use: https://www.spiedigitallibrary.org/terms-of-use 


\title{
Conference Committee
}

\author{
Symposium Chair
}

Thomas Becker, EADS Deutschland GmbH (Germany)

Symposium Cochairs

José Feliciano López, Universidad de Las Palmas de Gran Canaria (Spain)

Gerhard Krötz, University of Applied Sciences in Kempten (Germany)

Conference Chair

Ángel B. Rodríguez-Vázquez, AnaFocus (Spain) and Instituto de Microelectrónica de Sevilla-CNM-CSIC (Spain)

Conference Cochairs

Ricardo A. Carmona-Galán, Instituto de Microelectrónica de Sevilla-CNM-CSIC (Spain)

Gustavo Liñán-Cembrano, Instituto de Microelectrónica de Sevilla-CNM-CSIC (Spain)

\section{Program Committee}

Paolo Arena, Università degli Studi di Catania (Italy)

Gert Cauwenberghs, University of California, San Diego (United States)

Radu Dogaru, Universitatea Politehnica Bucuresti (Romania)

Antoine Dupret, Université Paris-Sud-XI (France)

José M. Ferrández, Universidad Politécnica de Cartagena (Spain)

Roman Genov, University of Toronto (Canada)

Giacomo Indiveri, ETH Zürich (Switzerland)

Pedro Julian, Universidad Nacional del Sur (Argentina)

Laura Maria Lechuga, CIN2 Research Center on Nanoscience and Nanotechnology (Spain)

Ramón Ruiz-Merino, Universidad Politécnica de Cartagena (Spain) Josep Samitier-Marti, Universitat de Barcelona (Spain)

Luis Serrano, Universidad Pública de Navarra (Spain)

Bertram Shi, Hong Kong University of Science and Technology (Hong Kong, China)

Ronald Tetzlaff, Johann Wolfgang Goethe-Universität Frankfurt am Main (Germany)

Fernando Vidal-Verdú, Universidad de Malaga (Spain)

Akos Zarandy, Computer and Automation Research Institute (Hungary) 
Session Chairs

$1 \quad$ Bioinspired Vision Chips and Algorithms

Paolo Arena, Università degli Studi di Catania (Italy)

2 Bioinspired Robot Control and Navigation

Gustavo Liñán-Cembrano, Instituto de Microelectrónica de Sevilla-CNM-CSIC (Spain)

3 Biological Models and Artificial Senses

Jean Tomas, Université Bordeaux I (France)

$4 \quad$ Biomedical Applications and Devices

Fernando Vidal-Verdú, Universidad de Malaga (Spain)

5 Biocompatible Materials Fabrication and Technologies

Ángel B. Rodríguez-Vázquez, AnaFocus (Spain) and Instituto de Microelectrónica de Sevilla-CNM-CSIC (Spain)

6 Neural Networks and Soft Computing

Ronald Tetzlaff, Technische Universität Dresden (Germany)

$7 \quad$ Microfluidics and Lab-on-a-Chip

Ricardo A. Carmona-Galán, Instituto de Microelectrónica de Sevilla-CNM-CSIC (Spain) 